\title{
A Toxicity Screening Approach to Identify Bacteriophage-Encoded Anti-Microbial Proteins
}

\author{
Ushanandini Mohanraj ${ }^{1,2}$, Xing Wan 1,3(0), Cindy M. Spruit ${ }^{1,4,+}$, Mikael Skurnik ${ }^{1,5}(1)$ \\ and Maria I. Pajunen $1, *(\mathbb{D}$ \\ 1 Department of Bacteriology and Immunology, Medicum, Human Microbiome Research Program, \\ Faculty of Medicine, University of Helsinki, 00290 Helsinki, Finland; \\ ushanandini.mohanraj@helsinki.fi (U.M.); xing.wan@helsinki.fi (X.W.); c.m.spruit@uu.nl (C.M.S.); \\ mikael.skurnik@helsinki.fi (M.S.) \\ 2 Department of Virology, Medicum, University of Helsinki, 00290 Helsinki, Finland \\ 3 Division Animal and Human Health Engineering, Kasteelpark Arenberg 21 - box 2462, 3001 Leuven, Belgium \\ 4 Laboratory of Microbiology, Wageningen University and Research, 6708 WE Wageningen, The Netherlands \\ 5 Division of Clinical Microbiology, Helsinki University Hospital, HUSLAB, 00290 Helsinki, Finland \\ * Correspondence: maria.pajunen@helsinki.fi; Tel.: +358-50-5677486 \\ + Present address: Department of Chemical Biology \& Drug Discovery, Utrecht Institute for Pharmaceutical \\ Sciences, Utrecht University, 3584 CG Utrecht, The Netherlands.
}

Received: 26 September 2019; Accepted: 12 November 2019; Published: 14 November 2019

\begin{abstract}
The rapid emergence of antibiotic resistance among many pathogenic bacteria has created a profound need to discover new alternatives to antibiotics. Bacteriophages, the viruses of microbes, express special proteins to overtake the metabolism of the bacterial host they infect, the best known of which are involved in bacterial lysis. However, the functions of majority of bacteriophage encoded gene products are not known, i.e., they represent the hypothetical proteins of unknown function (HPUFs). In the current study we present a phage genomics-based screening approach to identify phage HPUFs with antibacterial activity with a long-term goal to use them as leads to find unknown targets to develop novel antibacterial compounds. The screening assay is based on the inhibition of bacterial growth when a toxic gene is expression-cloned into a plasmid vector. It utilizes an optimized plating assay producing a significant difference in the number of transformants after ligation of the toxic and non-toxic genes into a cloning vector. The screening assay was first tested and optimized using several known toxic and non-toxic genes. Then, it was applied to screen 94 HPUFs of bacteriophage $\varphi$ R1-RT, and identified four HPUFs that were toxic to Escherichia coli. This optimized assay is in principle useful in the search for bactericidal proteins of any phage, and also opens new possibilities to understanding the strategies bacteriophages use to overtake bacterial hosts.
\end{abstract}

Keywords: HPUF; antibacterials; screening; bacteriophages; assay; toxic; $\varphi$ R1-RT

\section{Introduction}

The widespread appearance of drug-resistant bacterial isolates has a profound burden on health care [1]. Significant efforts have been made for the development of new and effective antibacterials to meet this challenge. A variety of natural compounds from different organisms have been found with antimicrobial activity [2]. When compared with natural product sources such as plants and animals, microorganisms are much more readily renewable and reproducible as a source. In recent years, viruses, especially bacteriophages, have emerged as a very potent source of antimicrobials [3].

Bacteriophages are viruses that infect bacterial cells [4]. Recently, there have been attempts to develop antibacterial agents from the bacteriostatic and bacteriolytic proteins encoded by 
bacteriophages, e.g., phage-encoded endolysins, VAPGH (virion-associated peptidoglycan hydrolases), polysaccharide depolymerases, and holins [3]. Bacterial cell lysis is a prerequisite for phage progeny release and dispersion for infection of new hosts. The majority of the phages lyse their bacterial hosts with the help of lytic enzymes by compromising the structural integrity of the peptidoglycan layer [5]. Hence, among the most promising alternatives to conventional antibiotics are phage-derived lytic enzymes.

Apart from these known lytic proteins, phages encode other gene products for which functions are not known. Such proteins of unknown function are called "hypothetical proteins of unknown function" (HPUF) [6]. Many hypothetical phage proteins are short polypeptides, and some are shown to inhibit the growth of the bacterial host [7-9]. There is an accumulation of a large number of proteins of unknown function in the databases due to the increasing gap between predicted phage gene sequences and their corresponding functions [10]. From what is already known, given their abundance and diversity, phage genomes could be packed with new genes involved in host inhibition. These would provide a rich source of leads for the development of novel antibacterials.

In the current study we describe a phage genomics-based screening approach of HPUFs to identify phage-encoded toxic products. Using this approach, we screened 94 HPUFs of Yersinia phage $\varphi$ R1-RT and identified four HPUFs toxic to Escherichia coli. Bacteriophage $\varphi$ R1-RT is distantly related to phage $\mathrm{T} 4$, and infects a number of Gram-negative Yersinia enterocolitica strains [11]. Y. enterocolitica, a member of the Enterobacteriaceae family, is a zoonotic pathogen causing human and animal enteric infections, including acute diarrhea, enterocolitis and terminal ileitis, and in rare cases sequelae such as reactive arthritis and erythema nodosum. When spread systemically, for example due to contaminated products used in blood transfusion, it can also cause fatal sepsis [12]. In the current work we demonstrated that using our screening approach, toxic proteins could be systematically identified among phage-encoded HPUFs. We further suggested that the toxic proteins in turn could be used to identify new bacterial targets for drug discovery.

\section{Materials and Methods}

\subsection{Bacterial Strains, Plasmids, Bacteriophages and Culture Conditions}

Bacterial strains, bacteriophages and plasmids used in this work are listed in Table 1. E. coli laboratory strain DH10B and DH5 $\alpha$ were used for cloning. Electrocompetent $E$. coli cells were prepared essentially as described previously [13]. LB agar plates were solidified with $1.5 \%(w / v)$ of agar. M9t minimal medium contained $3.4 \mathrm{mM} \mathrm{Na}_{2} \mathrm{HPO}_{4}, 2.2 \mathrm{mM} \mathrm{KH}_{2} \mathrm{PO}_{4}, 0.86 \mathrm{mM} \mathrm{NaCl}, 0.94 \mathrm{mM} \mathrm{NH} \mathrm{Cl}_{4} \mathrm{Cl}$ $0.2 \%(w / v)$ tryptone, $2.0 \mathrm{mM} \mathrm{MgSO}{ }_{4}, 0.10 \mathrm{mM} \mathrm{CaCl}_{2}$ and $3.0 \times 10^{-3} \mathrm{mM}$ vitamin $\mathrm{B} 1$ (Merck KGaA, Darmstadt, Germany). Media (agar, tryptone and yeast extract) were purchased from Neogen Food Safety, Lansing, MI, USA. When required, $100 \mu \mathrm{g} / \mathrm{mL}$ ampicillin (Amp; Sigma-Aldrich, St. Louis, MO, USA) was used.

Table 1. Bacterial strains, bacteriophages and plasmids used in the current study.

\begin{tabular}{ccc}
\hline Name & Description & Source \\
\hline E. coli strains & Used for HPUFs screening \\
DH10B & Used for pBAD30 cloning \\
DH5 $\alpha$ & NEB, USA \\
Bacteriophages & NEB, USA \\
कR1-RT & $\begin{array}{c}\text { Used as a template for amplifying all the } \\
\phi R \text {-RT encoded genes used in the } \\
\text { current study }\end{array}$ & \\
T4 & $\begin{array}{c}\text { Used as a template for amplifying regB } \\
\text { and ndd control genes }\end{array}$ & {$[11]$} \\
\hline
\end{tabular}


Table 1. Cont.

\begin{tabular}{|c|c|c|}
\hline Name & Description & Source \\
\hline \multicolumn{3}{|l|}{ Plasmids } \\
\hline pETSmt3- DCD-1L & $\begin{array}{l}\text { Used as template to amplify the } d c d-1 l \\
\text { control gene }\end{array}$ & [15] \\
\hline pU11L4 & $\begin{array}{l}\text { This plasmid consists of pUC19 with } \\
\text { a KpnI-PstI linker in the MCS and luxAB } \\
\text { genes under expression of ompF promoter } \\
\text { of YeO:3 at the SapI site (Figure S1. }\end{array}$ & This study \\
\hline$\phi X 174$ RF1 & $\begin{array}{l}\text { Used as template to amplify the lysE } \\
\text { control gene }\end{array}$ & Thermo Fisher Scientific, USA \\
\hline pBAD30 & $\begin{array}{l}\text { Plasmid with arabinose-inducible } \\
\text { promoter to express } \phi R 1-R T \text { HPUFs in } E \text {. } \\
\text { coli for confirmatory assay }\end{array}$ & [16] \\
\hline
\end{tabular}

\subsection{Mass Spectrometry (MS) Data Analysis of Phage Proteins}

The LC-MS/MS experiments were carried out at the Proteomics Unit, Institute of Biotechnology, University of Helsinki, using the protocols described elsewhere [17]. The obtained peptide sequences from the MS were compared simultaneously against the phage and bacterial (Y. enterocolitica serotype O:3 strain Y11 - Accession number FR729477.2) protein sequences. From the LC-MS/MS analysis, proteins which fulfilled the inclusion criteria ( $>2$ unique peptides and/or $>5 \%$ coverage) were considered for annotation as phage particle associated proteins. The hypothetical proteins that were identified in the LC-MS/MS data were re-annotated to hypothetical structural proteins in the $\varphi$ R1-RT genome sequence under the accession number HE956709.

\subsection{Comparative Protein Analysis of Hypothetical Proteins of Unknown Function (HPUFs) and Genome Annotation}

To re-evaluate the putative functions of the HPUFs, similarity searches were performed using the NCBI BLASTp tool [18] against the non-redundant protein database to confirm the absence of any HPUF homologs with known functions. Proteins with low query coverage $(<50 \%)$ or low sequence identity $(<20 \%)$ were not considered, while proteins with high sequence identities $(>40 \%)$, and low $E$-value $(<0.005)$ were considered as close homologs. In addition to BLASTp, Hidden Markov Models (HMMs)-based HHpred tool [19] was used, which provided domain-based similarity searching for the query sequence. HHpred tool search was performed against a database of PDB, SCOP and Pfam to identify the putative domains and functions of individual HPUFs.

\subsection{PCR Amplification of Control and Hypothetical Proteins of Unknown Function Genes for Cloning}

The sequences of the genes amplified in the current study were retrieved from GenBank. All the primers were designed manually according to [20] and sent to Metabion (Germany) for synthesis. Supplementary Tables S1 and S2 list the sequences of all the primers that were designed and used in the present study.

Reactions were performed either in $0.2 \mathrm{~mL}$ thin walled PCR tubes (4titude ${ }^{\circledR}$ Ltd., Wotton, UK), in $50 \mu \mathrm{L}$ volumes containing $10 \mathrm{ng}$ of DNA template for single tube reactions, or in 96 well microtiter plate format using $30 \mu \mathrm{L}$ volumes containing $5 \mathrm{ng}$ of DNA template. For each reaction, $0.5 \mu \mathrm{M}$ of primers, $0.2 \mathrm{mM}$ of dNTP mix (Thermo Fisher Scientific, Waltham, MA, USA), 10 or $6 \mu \mathrm{L}$ of $5 \times$ Phusion Buffer and $0.02 \mathrm{U} / \mu \mathrm{L}$ Phusion DNA Polymerase (Thermo Fisher Scientific, Waltham, MA, USA) were added. The PCR cycling included an initial denaturation at $98{ }^{\circ} \mathrm{C}$ for $30 \mathrm{~s}$ followed by 30 cycles, each consisting of $10 \mathrm{~s}$ at $98^{\circ} \mathrm{C}, 30 \mathrm{~s}$ at Tm and $30 \mathrm{~s}$ at $72{ }^{\circ} \mathrm{C}$ for extension, followed by a final extension step at $72{ }^{\circ} \mathrm{C}$ for $10 \mathrm{~min}$. The Tm, annealing temperature, was calculated for each primer pair using the web-tool at: https://www.thermofisher.com/in/en/home/brands/thermo-scientific/ molecular-biology/molecular-biology-learning-center/molecular-biology-resource-library/thermoscientific-web-tools/tm-calculator.html. The PCR products were kept on hold, at $4{ }^{\circ} \mathrm{C}$, until further 
processing. When using the phage particles directly as a template for PCR, $2 \mu \mathrm{L}$ ( $\sim 60 \mathrm{ng}$ ) of phage particle suspension was used as a template with initial denaturation at $98^{\circ} \mathrm{C}$ for $3 \mathrm{~min}$. The rest of the PCR cycle steps were as described above.

\subsection{Cloning}

The toxic and non-toxic control genes, and the $\varphi$ R1-RT HPUFs genes were cloned into the multiple cloning site of the pUC19-based vector pU11L4 using the NotI and NcoI restriction sites (Thermo Fisher Scientific, Waltham, MA, USA) (Figure S1). Whenever an internal NcoI site was present in any of the HPUF-genes, the NheI (Thermo Fisher Scientific, Waltham, MA, USA) digestion was used instead.

All the ligation reactions in the present study were prepared in final volumes of $15 \mu$ l consisting of $75 \mathrm{ng}$ of vector with a vector to insert ratio 1:3, 1.5 $\mu$ l of 10X T4 Ligation Buffer (NEB, Ipswich, MA, USA) and $3 \mathrm{U}$ of T4 DNA Ligase (NEB, Ipswich, MA, USA). The reactions were incubated overnight at $16{ }^{\circ} \mathrm{C}$, heat inactivated at $65^{\circ} \mathrm{C}$ for $20 \mathrm{~min}$ and stored at $-20{ }^{\circ} \mathrm{C}$ until further use.

Transformations were done by electroporation with the exponential decay pulses generated by a Gene Pulser ${ }^{\mathrm{TM}}$ apparatus (Bio-Rad Laboratories, Hercules, CA, USA) set at $25 \mu \mathrm{F}$ and $2.5 \mathrm{kV}$ for $2 \mathrm{~mm}$ cuvettes. The competent cells were first thawed on ice. Then $45 \mu \mathrm{L}$ of cells and $2 \mu \mathrm{L}$ of ligated DNA solution were transferred to a cold, $1.5 \mathrm{~mL}$ polypropylene tube; the suspension was mixed by flicking the tube. The cell/DNA mixture was then added to the chilled cuvettes, and the appropriate pulse applied. Following the pulse, the cells were immediately removed from the electrodes and mixed into $950 \mu \mathrm{L}$ of SOC outgrowth medium (SOC: $2 \%$ Bacto tryptone, $0.5 \%$ Bacto yeast extract, $10 \mathrm{mM}$ $\mathrm{NaCl}, 2.5 \mathrm{mM} \mathrm{KCI}, 10 \mathrm{mM} \mathrm{MgCl} 2,10 \mathrm{mM} \mathrm{MgSO}_{4}, 20 \mathrm{mM}$ glucose). The samples were incubated, with shaking at $225 \mathrm{rpm}$, for $1 \mathrm{~h}$ at $37^{\circ} \mathrm{C}$. After this, the cells were plated on LB-agar containing selective antibiotics to screen for transformants.

\subsection{Screening for Toxic Hypothetical Proteins of Unknown Function Genes}

All the 94 hypothetical genes were PCR amplified, restricted and ligated as previously described. After electroporation, the transformation mix was plated on the LB-Ampicillin agar plates. The plates were kept at $37^{\circ} \mathrm{C}$ overnight and next day colonies were counted using a colony counter. Each screening experiment included the HPUF genes and at least one non-toxic control gene. Colony forming units (CFU) were normalized to the CFU of the non-toxic control gene in an individual experiment and expressed as relative CFUs.

\subsection{Confirmation of Protein Toxicity}

Potentially toxic $\varphi$ R1-RT HPUFs that were selected in the initial screening assay and control genes g150 and $l y s E$ were cloned into the pBAD30 vector [16] using EcoRI and XbaI (Thermo Fisher Scientific, Waltham, MA, USA) restriction sites. Whenever an internal $\mathrm{XbaI}$ restriction site was present, cloning was performed with PaeI, whereas EcoRI was replaced with KpnI (Thermo Fisher Scientific, Waltham, MA, USA) instead. The plasmids were purified and the presence of the correct inserts was confirmed by sequencing at the Institute for Molecular Medicine Finland Technology Centre Sequencing Unit [21]. The plasmids were electroporated into electrocompetent $E$. coli DH5 $\alpha$. Three single colonies per gene were inoculated into $1 \mathrm{~mL}$ LB medium supplemented with $0.2 \%(w / v)$ glucose and $100 \mu \mathrm{g} / \mathrm{mL}$ ampicillin (Amp; Sigma-Aldrich, St. Louis, MO, USA) in a $2 \mathrm{~mL}$ Eppendorf tube and incubated overnight at $37^{\circ} \mathrm{C}$ with shaking at $160 \mathrm{rpm}$. The following day, the bacteria were collected by centrifuging for $5 \mathrm{~min}$ at $8000 \mathrm{rcf}$ and the medium was replaced with $1 \mathrm{~mL}$ minimal medium M9t. Afterwards, M9t medium was supplemented with $100 \mu \mathrm{g} / \mathrm{mL}$ Amp and either $0.2 \%(w / v)$ glucose or $0.2 \%(w / v)$ arabinose was inoculated with $1 \%$ inoculum of washed bacterial cells. The bacterial dilutions were transferred to Bioscreen Honeycomb 2 plates in triplicate $(300 \mu \mathrm{L} /$ well). The OD at $600 \mathrm{~nm}$ was measured every hour for $16 \mathrm{~h}$ using the Bioscreen C MBR (Oy Growth Curves Ab Ltd., Helsinki, Finland). The plate was shaken continuously with high amplitude and normal speed. Shaking was stopped $10 \mathrm{~s}$ before measuring and measuring was started immediately. Average values over the triplicate measurements 
were taken. The overall average values and standard deviations were calculated over the three biological replicates.

\subsection{Structure Prediction of Toxic HPUFs}

Modeling and secondary structure prediction of the hypothetical proteins was performed using the online Phyre2 modelling server accessed on 15.07.2019 [22].

\subsection{Statistics}

Graphical representations and statistical analysis were performed using Origin 7.5 Software (OriginLab Corp., Northampton, MA, USA) with data presented as the means \pm standard deviation (SD).

\section{Results}

\subsection{Elimination of Hypothetical Proteins of Unknown Function (HPUFs) by LC-MS/MS Analysis of Phage} Particle Proteomes

In the $\varphi$ R1-RT genome (HE956709), 129 predicted genes were annotated to encode HPUFs. To exclude the structural genes from the screening approach the phage particle structural proteins of the $\varphi$ R1-RT phage were identified using the LC-MS/MS proteomics data. Altogether, 78 phage proteins that fulfilled the inclusion criteria were identified in the LC-MS/MS analysis (Supplementary Table S3). These included 21 proteins earlier annotated as HPUFs, and based on the LC-MS/MS data they were re-annotated as putative structural proteins and eliminated from the toxic protein screening approach (Supplementary Table S4).

\subsection{Further Elimination of Hypothetical Proteins of Unknown Function (HPUFs) by BLASTp and HHpred Analyses}

The remaining 108 HPUFs were subjected to BLASTp and HHpred searches to identify other HPUFs with predictable functions to be eliminated from the screening (Supplementary Table S5).

Of the 108 hypothetical proteins, Gp019, Gp138, and Gp255, had no significant hits in the BLASTp search. On the other hand, nine were found to show significant similarity to proteins of known function; of these, six were involved in nucleotide metabolism, and three as phage structural proteins. All the others had hypothetical proteins as major BLASTp hits. Putative conserved domains were detected for 21 proteins. Out of the 21, 7 had domains that belonged to the protein families of unknown function. A transmembrane helix was detected for Gp076.

In the HHpred search only 39 proteins had significant hits. One transmembrane helix was detected in Gp076 and Gp094, and two transmembrane helices were detected in Gp105 and Gp137. In addition, coiled-coil segments were detected in Gp087, Gp098, and Gp143.

In summary, based on the BLASTp and HHpred searches, altogether 14 HPUFs were further removed from the true HPUFs; eight (Gp139, Gp143, Gp183, Gp184, Gp185, Gp251, Gp038, and Gp213) that were similar to other known structural proteins, five (Gp039, Gp047, Gp057, Gp075, and Gp179) that had domains similar to proteins required for DNA replication, transcription, translation or nucleotide metabolism, and one (Gp107) that was similar to rI lysis inhibition regulator membrane protein of Yersinia phage vB_YenM_TG1. Hence, the remaining 94 HPUFs were selected to be screened for toxic ones.

\subsection{Ligations of Toxic and Non-Toxic Control Genes Show Differences in Plating Efficiency}

Based on the obtained proteomics data the $g 121, g 178, g 246$ and $g 150$ genes of $\varphi$ R1-RT were selected as non-toxic controls, and based on a literature search, the $n d d, d c d-1 l, l y s E$ and regB genes were selected as toxic controls. When the E. coli DH10B cells were transformed with mixtures containing plasmid pU11L4 ligated with PCR fragments of the control genes encoding toxic and non-toxic proteins, significant differences were observed between the number of transformants obtained (Figure 1). 
The regB and $d c d-1 l$ genes were observed to be more toxic to $E$. coli cells as compared to $l y s E$ and $n d d$. We also observed that the non-toxic control gene $g 121$ resulted in lower transformation efficiency as compared to the other non-toxic control genes. Based on the difference in the transformation efficiencies observed between the different control toxic and non-toxic genes, an assay was developed to screen for toxic HPUF genes.

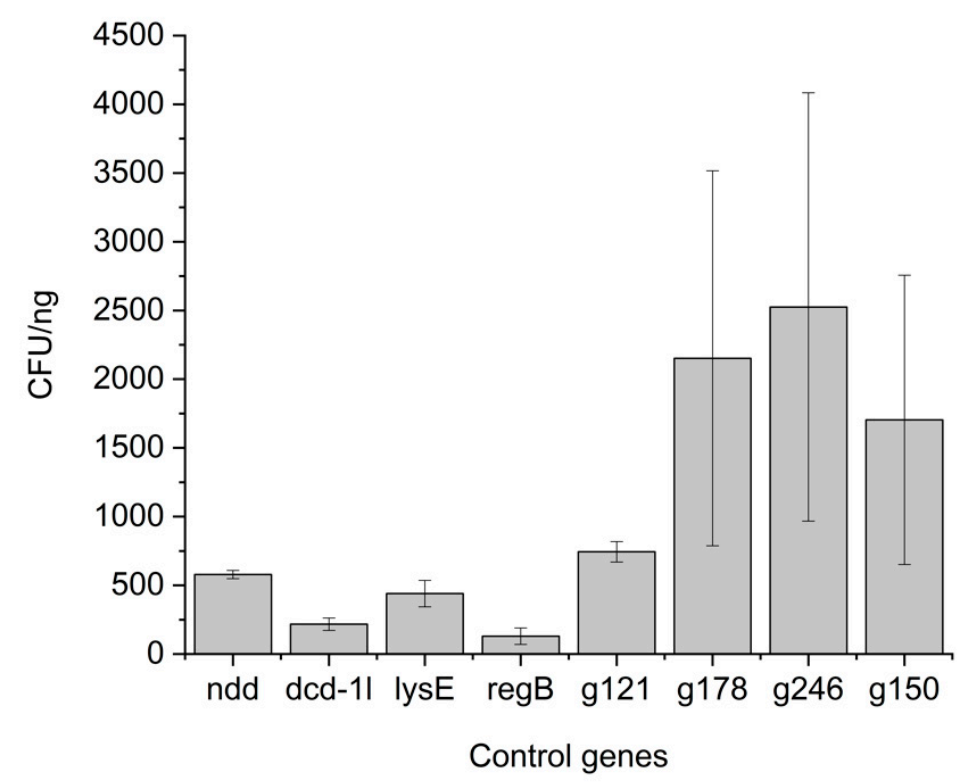

Figure 1. The differences in transformation efficiencies of ligation mixtures of known toxic and non-toxic genes. The bars indicate mean $\pm S D$ in the CFU/ng values of two different replicates from two different electroporations for each sample.

\subsection{Eight Potential Toxic Protein Hits Identified from an Initial CFU-Based Screening Approach}

After successful amplification of all the 94 HPUF genes, they were digested for ligation by appropriate restriction digestions, and ligated with the pU11L4 plasmid. The obtained ligation mixtures were transformed into E. coli DH10B cells. CFUs were counted for all the 94 HPUFs and relative CFU values were calculated (Supplementary Table S6). Figure 2A-C represents relative CFU values for all the 94 HPUF genes normalized to the non-toxic control gene included in each individual transformation experiment. Based on the observation of the CFU values of the control toxic genes and their difference with the non-toxic genes, a cut-off value of 0.4 ( $40 \%$ of the non-toxic control) was chosen for the screening by plating assay. HPUFs displaying a relative CFU value equal or lower than 0.4 upon transformation were considered toxic and further analyzed (Figure 2A,B). When considering g121 as a non-toxic control, instead of considering hits below a relative CFU value of 0.4 , hits below relative CFU 0.5 were considered since $g 121$ gave consistently lower CFU values as compared to the other non-toxic controls (Figure 2C). From this initial screening, eight potential hits toxic to E. coli cells were identified. 


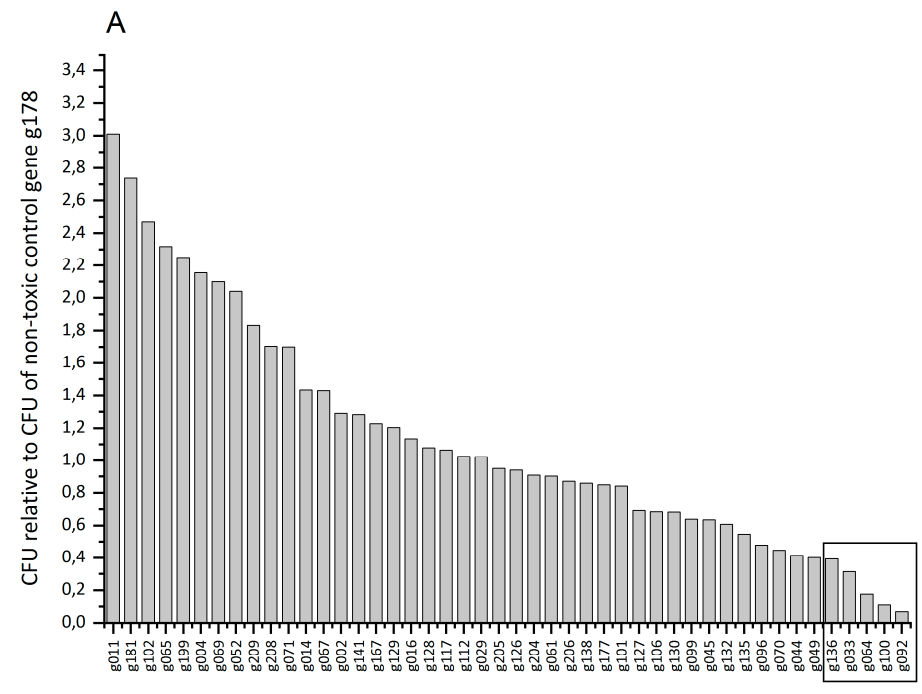

фR1-RT HPUF genes

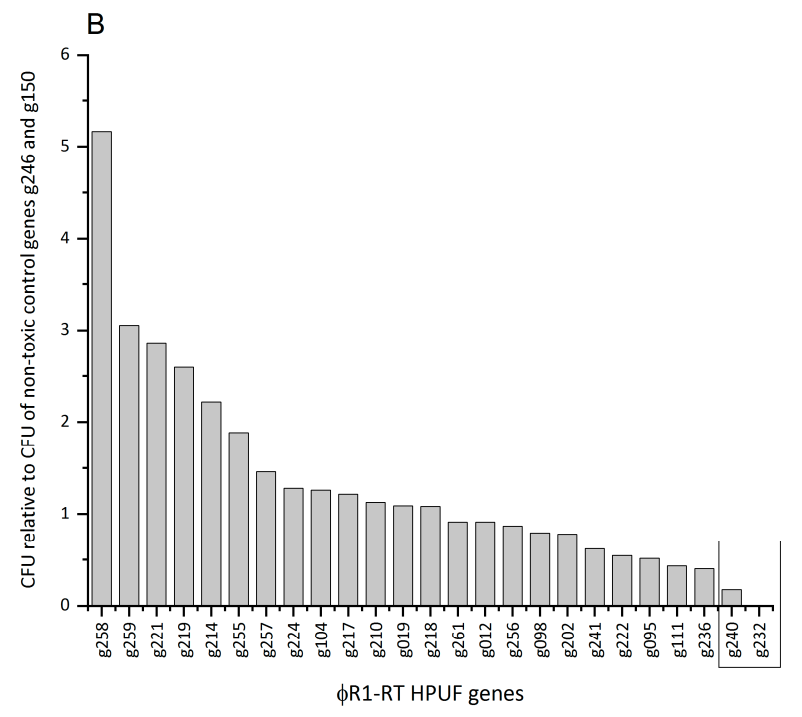

C

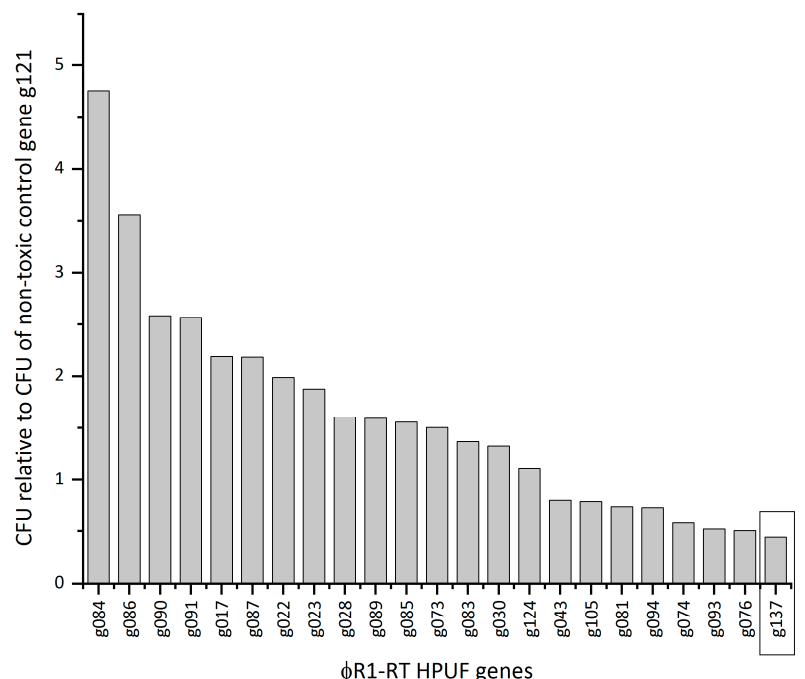

Figure 2. Relative CFU results of the 94 HPUF genes from the screening by plating assay. The plating efficiencies were normalized relative to the control genes $g 178$ (A), g246 and $g 150$ (B) or $g 121$ (C). 


\subsection{Toxic Protein Hits Gp064, Gp136, Gp137 and Gp232 Inhibit the Growth of E. coli Cells}

To confirm the toxic hits obtained in the initial screening, the genes of the eight potentially toxic HPUFs were cloned into pBAD30 under the control of the arabinose promoter $\mathrm{P}_{\mathrm{BAD}}$. In the presence of arabinose, the E. coli $\mathrm{DH} 5 \alpha$ bacteria carrying the plasmids will express the HPUF gene, whereas in the presence of glucose, the expression is repressed. To avoid the possible influence of glucose present in a rich LB medium, a semi-defined minimal medium M9t was used. The recombinant bacteria with 8150 , the known non-toxic control gene, showed similar growth both under inducing and repressing conditions. This was in contrast to the recombinants expressing the toxic control gene, lysE, or four of the eight toxic HPUF hits, i.e., g064, g136, g137 and g232. These showed retarded growth in the presence of arabinose (Figure 3) and were thus regarded as toxic when expressed in E. coli.
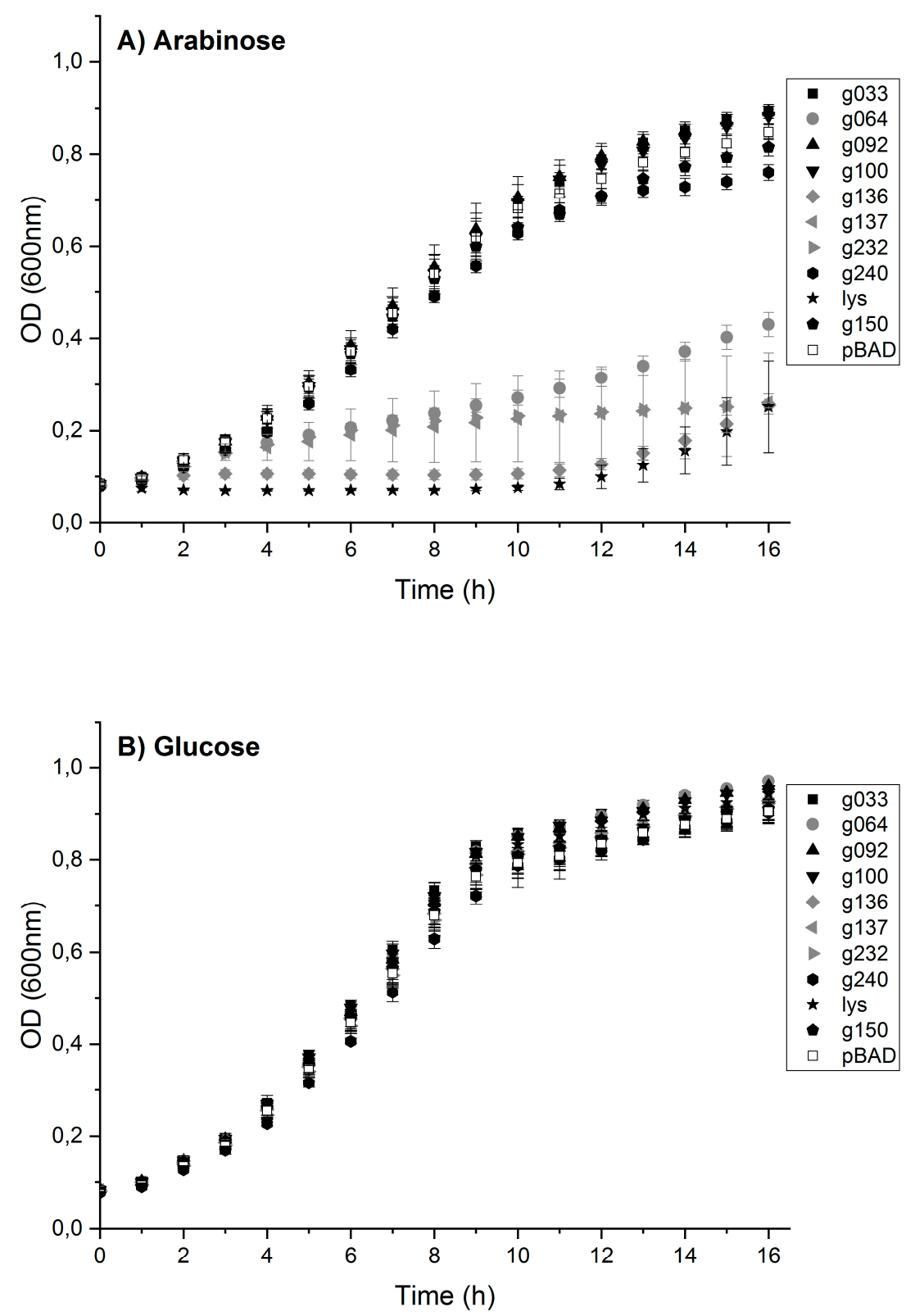

Figure 3. Growth curves of recombinant E. coli expressing phage proteins under the control of arabinose inducible promoter in pBAD30 in minimal media M9t supplemented with (A) arabinose for gene expression or $\mathbf{( B )}$ glucose for gene repression. 


\subsection{Modelling Studies}

The Phyre2 software was applied to model putative functional domains and/or secondary structures for the toxic HPUFs Gp064, Gp136, Gp137 and Gp232. Except for Gp232, the models produced had very low confidence levels. Interestingly, for Gp232, a stretch of 92 residues could be reliably modelled with $98.7 \%$ confidence and $32 \%$ identity using the homing endonuclease (PDB ID: 3R3P) from Bacillus phage 0305phi8-36 as the template (Figure 4). In Phyre2, the query and the template sequence identity should be at least $30-40 \%$ and the confidence level $>90 \%$, for a reliable model [22]. Additionally, only one transmembrane helix was predicted for Gp136, and two transmembrane helices for Gp137 (Figure 5).

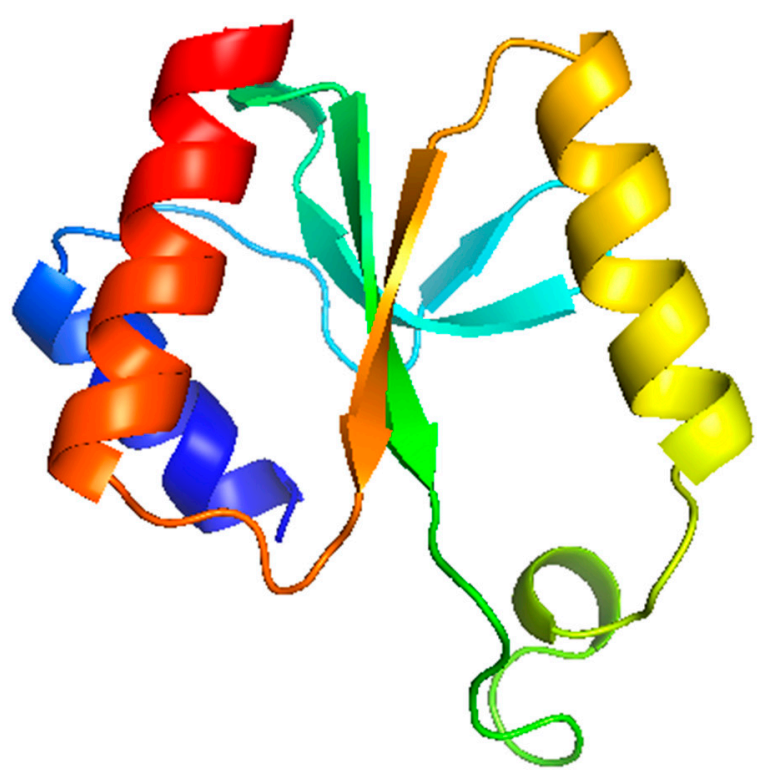

Figure 4. The predicted tertiary structure for the 92 residues ( 245 aa- 344 aa) of Gp232. The image is colored by rainbow $\mathrm{N} \rightarrow \mathrm{C}$ terminus.

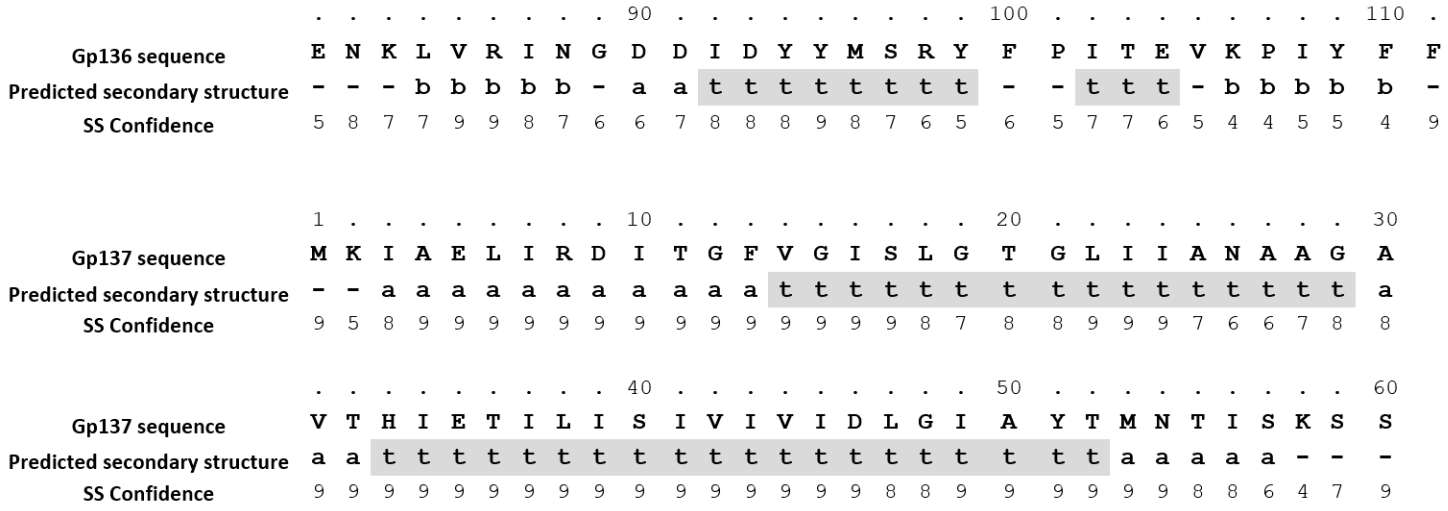

Figure 5. Predicted transmembrane helices for Gp136 and Gp137. a-Alpha helix, b-beta strand, t-TM helix. Numbers 0-9 represent secondary structure prediction confidence by Phyre2.

\section{Discussion}

With the accumulation of sequence data on thousands of new bacteriophage genomes annually we still face the fact that a majority of the predicted genes encode proteins (i.e., HPUFs) either with no similarity to any proteins in the databases, or similarity to proteins with unknown functions $[23,24]$. Hence, toxicity screening of these HPUFs, and subsequent identification of their mode of action will likely reveal novel targets for antibacterial agents. It is unlikely that the expression of any toxic 
phage product would be continuous throughout the phage infection cycle, instead it likely occurs at a certain phase of the infection [25]. In the current study, a phage genomics-based screening method was designed, and as a proof of principle, the HPUFs of phage $\varphi$ R1-RT were screened for toxic ones. The phage $\varphi$ R1-RT genome (HE956709) was $168 \mathrm{~kb}$ in size with 262 predicted genes [11]. Based on similarity searches, putative functions were assigned to only 133 gene products $(50.7 \%)$ of phage $\varphi \mathrm{R} 1-\mathrm{RT}$, thus the remaining 129 gene products were annotated as HPUFs.

A proteomic analysis carried out on purified phage particles has allowed for the assignment of some of the HPUFs as structural proteins [26]. From an antibacterial point of view, our interest lies in the phage proteins that would inhibit the host bacteria upon expression, and hence the structural proteins would not belong to this group of HPUFs. Proteins that are not identified in the LC-MS/MS analysis are likely the proteins whose expression takes place in the host and are not part of the virion. Hence LC-MS/MS analysis of purified phage particles directly measures peptides arising from the structural phage proteins. However, there is a possibility that some of the identified proteins are carried over from the lysate. A phage lysate is obtained by infecting host bacteria with the phage and letting the phage lyse the cells [27]. It cannot be excluded that some bacterial proteins will be co-isolated with the phage particles and identified due to the high sensitivity of the method. Hence the obtained peptide sequences from the LC-MS/MS were compared simultaneously against not only the phage but also the host bacterial (Y. enterocolitica serotype O:3 strain Y11 - FR729477.2) protein sequences as well. On the other hand, some phage structural proteins might not be detected due to their low abundance in the phage particles. Some of these were identified using BLASTp and/or HHpred analysis. HHpred is known for its sensitivity in detecting remote protein homology, for structure predictions, and it uses a pairwise comparison of profile HMMs [19].

From the initial LC-MS/MS data (Supplementary Table S3), it was observed that not all of the phage particle-associated proteins were structural proteins e.g., Gp235 was identified as thymidylate synthase, Gp055, as DNA polymerase, Gp003, as DenB, the DNA endonuclease IV, Gp233, as ribonucleotide reductase of class Ia, Gp031, as dCTP pyrophosphatase, and Gp082, as thioredoxin. While it is possible that these proteins are truly associated with the phage particles, they may also possess physical properties that make them more prone to being co-isolated with phage particles during the purification process, or that they might display non-specific binding to the capsid proteins. Some more complex phages package their RNA polymerase into the phage head and eject it into the host cell along with the phage DNA in order to facilitate the transcription of early phage genes [28], supporting the true association. This makes sense as upon infection the first step in the phage life cycle is transcription requiring DNA-dependent RNA polymerase and its associated factors [29]. Furthermore, the bacteriophage T4 gene 42 encodes the dCMP hydroxymethylase, an enzyme unique to the deoxyribonucleotide metabolism of T-even bacteriophages [30]. After eliminating all putative structural HPUFs and those required for transcription or nucleotide metabolism with MS analysis and BLASTp and HHpred, 94 HPUF genes were considered for screening.

In one of the earlier studies involving screening of phage genomes for S. aureus growth inhibitory gene products, phage genes were cloned under the control of an arsenite-inducible promoter [7]. Using this method, 31 novel toxic protein families were found from these phage genomes. In their approach, they screened all the predicted phage genes and expression cloned each one of them for toxic protein identification. Another screening approach that has been previously reported for the identification of inhibitory gene products involved the construction of genomic libraries of phages and the identification of inhibitory genes [8,31]. In both approaches, there was a possibility for the presence of structural genes and genes that were involved in the nucleotide metabolism, in the screening process. As these gene products are likely non-toxic their inclusion in the screen would be a waste of resources and effort.

The incomplete repression of promoter combined with the effects of high-copy-number plasmids and transcriptional read-through, would result in the expression of a toxic protein and the killing of the bacterial host [32]. In line with this, we observed that ligation of the toxic genes regB, $n d d$, 
lysE and $d c d-1 l$ into pU11L4 plasmid resulted in a reduced number of transformants as compared to the non-toxic genes $g 150, g 178$, g246 and g121. Based on this we designed our screening approach such that we cloned the ninety-four HPUF genes into pU11L4 plasmids and screened for those that resulted in lower transformation efficiencies as compared to the non-toxic control genes. Furthermore, we confirmed the toxicities of the genes from the initial screening through an arabinose inducible expression system. Our confirmatory experiments on eight HPUF genes suggested a true-positive predictive rate of $50 \%$, which meant that half of the genes predicted to be toxic based on initial screening information were found to inhibit the growth of bacteria under a controlled expression.

From the inter-assay variability experiment (Figure S2), we observed that the coefficient of variance for observed transformation efficiencies from two separate batches of ligation for genes g033, g064, $g 092, g 100$ and $g 232$ was more that $20 \%$. From the pBAD assay, three of these genes $g 033, g 092$ and $g 100$ were not truly toxic. Hence, by reducing the assay-to-assay variations, the true positive prediction rate could be improved in our screening approach. The variation in the plating efficiency of a single gene could be attributed to experiment-to-experiment variation between setting up the ligation mixtures, the electroporation and plating efficiencies. In our screening approach (Figure 1), we calculated the transformation efficiencies per nanogram of ligation mixture as opposed to the use of purified plasmids. Though use of the ligation mix directly in the screening process saves time, there is the possibility of the ligation mixtures containing widely different absolute numbers of successfully ligated plasmids with inserts to be electroporated into the bacteria. Despite the use of the same ligation conditions and insert to vector ratio for all the experiments, the ligation efficiencies could vary, resulting in different amounts of linear and circular plasmids. Furthermore, variation could also arise during the spreading and growth of the bacteria on the plates, resulting in a different amount of CFUs per plate for the same volume of transformed cells. In future experiments, therefore, we will use ligation and plating replicates in the initial screening assay to minimize variation and reduce false positive hits.

The genes encoding endolysin (g122) and holin ( $(253)$ were previously identified from the $\varphi$ R1-RT genome [11]. Additionally, the gene $g 252$ product showed similarity to a holin. As the endolysins and holins of many phages have demonstrated antibacterial activity [3], we also tested the $\varphi$ R1-RT endolysin and holin encoding genes in the screening by plating assay for their toxicity towards E. coli. To our surprise we did not see the expected reduction in the plating efficiency with any of the genes (Supplementary Table S7). A plausible explanation for this is that T4-like endolysins and holins are not active alone, as is the case in the closely related T4 [33]: "In the absence of either the lysozyme or the holin, lysis does not occur". T4 holin (the gene $t$ product) is unlike many other holins not immediately toxic but requires its own endolysin for cell lysis to occur. Analogously, a lysis gene cassette of Burkholderia pseudomallei bacteriophage ST79 contains four genes, and minimally for lysis to occur, the expression of the peptidase and holin encoding genes is required, and only moderate lysis was observed when holin alone was expressed [34]. Similar dependence of a phage holin on cognate endolysin was observed for Streptococcus suis phage SMP, where the holin alone caused only weak lysis but with endolysin strong lysis [35]. In our screening approach we did not detect these interaction-dependent toxic phage products including proteins that require other phage-encoded proteins for proper folding or for other forms of post-translational modification.

Based on Phyre2 modelling, an endonuclease domain was predicted for Gp232. Endonucleases and other nucleic acid cleaving enzymes belong to a large and extremely diverse family of proteins displaying little sequence similarity though they retain a common core fold responsible for cleavage. Some endonucleases include different types of $\mathrm{Zn}$-binding and DNA-binding domains [36]. The confidence levels of models of all the other toxic hits were low and hence were not reliable. Therefore, our current data does not indicate any putative function for these hits and hence further biochemical studies are required. On the other hand, this may indicate that these hits might constitute a completely novel class of antibacterial proteins or peptides without homology to any currently known protein.

In a previous study [9], affinity purification mass spectrometry was used to identify phage proteins binding to specific bacterial targets. The bacterial targets were selected based on the hypothesis 
that phages could affect important metabolic proteins, involved in general transcriptional regulation, post-transcriptional regulation, fatty acid biogenesis, cell division, and energy household. Furthermore, many phages are known to encode proteins that interact with the RNA and DNA polymerases of their hosts to either inhibit or redirect bacterial transcription and genome replication. Based on this approach eight proteins that are toxic to Pseudomonas aeruginosa were identified, of which four were also toxic to E. coli. Though this method is efficient in identifying toxic proteins along with their bacterial targets, this approach limits the identification of toxic proteins interacting with novel bacterial targets.

The results from our study are aimed at forming the basis for phage-host interaction studies, which has a great potential to reveal novel antibacterial mechanisms and molecular targets to treat bacterial infections. Our current fast screening approach for new toxic proteins should be generally applicable to phages infecting relatively closely related bacterial hosts, such as Enterobacteriaceae species. The toxic phage proteins are not likely to act as such to kill bacteria extracellularly as they cannot penetrate the cell walls and membranes. Therefore, investigations on the molecular level interactions between the toxic hits and the host bacteria are required to expose their targets, which in turn could be used to screen small molecule libraries for inhibitors. We can think of two possible approaches to identify the targets. First, the phage toxin should be purified to be used as a bait in fishing out the target. As the expression of the protein in E. coli, due to its toxicity, may be difficult, yeast or plant cells could be used instead, or ultimately, in vitro translation could be applied. Second, a genetic approach could be used where spontaneous toxin-insensitive mutants would be isolated and the target identification would be carried out by whole genome sequencing of the mutants.

Although only four toxic hits were identified among the $94 \varphi$ R1-RT HPUFs, we are confident that the genomics-based screening of toxic phage proteins, to be followed by identification of the respective phage-host interactions, as well as structural studies of individual interacting partners has the potential to generate big data for the contemporary drug industry.

\section{Conclusions}

Although it has been stated that functional elucidation of antibacterial phage proteins could be a powerful tool in target identification and drug discovery, only limited progress has been made in the last decade. Our study presents a screening approach to identify toxic phage HPUFs based on their ability to inhibit bacterial growth upon intracellular expression in the host bacteria. In the current study, four toxic HPUFs against $E$. coli were identified. The screening assay allows us to systematically examine and identify toxic genes from hypothetical phage proteins that, as far as we know, are not currently pursued by the industry as targets for antibiotic development.

Supplementary Materials: The following are available online at http://www.mdpi.com/1999-4915/11/11/1057/s1, Table S1. Primers used to amplify the non-toxic and toxic control genes, Table S2. Primers used to amplify the HPUF genes from $\varphi$ R1-RT phage, Table S3. $\varphi$ R1-RT structural proteins identified by LC-MS/MS analysis, Table S4. Hypothetical proteins annotated as structural proteins from the LC-MS/MS analysis, Table S5. BLASTp and HHpred analysis results for the HPUFs, Table S6. CFU counts of HPUFs and relative CFU of HPUFs to the CFU of non-toxic control genes in that batch of electroporations, Table S7. CFU values for $\varphi$ R1-RT encoded holins and endolysin relative to CFU of control non-toxic gene g119, Figure S1. Map of the plasmid construct pU11L4 with $K p n I-P s t I$ linker and $l u x A B$, Figure S2. Inter-assay variability of the screening assay.

Author Contributions: Conceptualization: U.M., M.S., M.I.P. Investigation: U.M., X.W., C.M.S., M.I.P. Methodology: U.M., X.W., M.S., M.I.P. Writing-Original draft preparation: U.M. Writing—Review and editing: U.M., X.W., C.M.S., M.S., M.I.P.

Funding: This research was funded by the Academy of Finland grant number 288701 to M.S. X.W. was funded by the Ella and Georg Ehrnrooth Foundation with a personal grant. Open access funding provided by University of Helsinki.

Acknowledgments: The authors would like to thank Alli Hietikko for her help with preparing electrocompetent cells.

Conflicts of Interest: The authors declare no conflicts of interest. 


\section{References}

1. Prestinaci, F.; Pezzotti, P.; Pantosti, A. Antimicrobial resistance: A global multifaceted phenomenon. Pathog. Glob. Health 2015, 109, 309-318. [CrossRef]

2. Newman, D.J.; Cragg, G.M. Natural products as sources of new drugs over the last 25 years. J. Nat. Prod. 2007, 70, 461-477. [CrossRef]

3. Roach, D.R.; Donovan, D.M. Antimicrobial bacteriophage-derived proteins and therapeutic applications. Bacteriophage 2015, 5, e1062590. [CrossRef] [PubMed]

4. Abedon, S.T.; Thomas-Abedon, C.; Thomas, A.; Mazure, H. Bacteriophage prehistory: Is or is not Hankin, 1896, a phage reference? Bacteriophage 2011, 1, 174-178. [CrossRef] [PubMed]

5. Young, R. Bacteriophage lysis: Mechanism and regulation. Microbiol. Rev. 1992, 56, 430-481. [PubMed]

6. Shahbaaz, M.; Hassan, M.I.; Ahmad, F. Functional annotation of conserved hypothetical proteins from Haemophilus influenzae Rd KW20. PLoS ONE 2013, 8, e84263. [CrossRef] [PubMed]

7. Liu, J.; Dehbi, M.; Moeck, G.; Arhin, F.; Bauda, P.; Bergeron, D.; Callejo, M.; Ferretti, V.; Ha, N.; Kwan, T.; et al. Antimicrobial drug discovery through bacteriophage genomics. Nat. Biotechnol. 2004, 22, 185-191. [CrossRef]

8. Shibayama, Y.; Dabbs, E.R. Phage as a source of antibacterial genes: Multiple inhibitory products encoded by Rhodococcus phage YF1. Bacteriophage 2011, 1, 195-197. [CrossRef]

9. Van den Bossche, A.; Ceyssens, P.J.; De Smet, J.; Hendrix, H.; Bellon, H.; Leimer, N.; Wagemans, J.; Delattre, A.-S.; Cenens, W.; Aertsen, A.; et al. Systematic identification of hypothetical bacteriophage proteins targeting key protein complexes of Pseudomonas aeruginosa. J. Proteome Res. 2014, 13, 4446-4456. [CrossRef]

10. Klimke, W.; O’Donovan, C.; White, O.; Brister, J.R.; Clark, K.; Fedorov, B.; Mizrachi, I.; Pruitt, K.D.; Tatusova, T. Solving the problem: Genome annotation standards before the data deluge. Stand. Genom. Sci. 2011, 5, 168-193. [CrossRef]

11. Leon-Velarde, C.G.; Happonen, L.; Pajunen, M.; Leskinen, K.; Kropinski, A.M.; Mattinen, L.; Rajtor, M.; Zur, J.; Smith, D.; Chen, S.; et al. Yersinia enterocolitica-specific infection by bacteriophages TG1 and $\phi$ R1-RT is dependent on temperature-regulated expression of the phage host receptor OmpF. Appl. Environ. Microbiol. 2016, 82, 5340-5353. [CrossRef] [PubMed]

12. Fàbrega, A.; Vila, J. Yersinia enterocolitica: Pathogenesis, virulence and antimicrobial resistance. Enferm. Infecc. Microbiol. Clin. 2012, 30, 24-32. [CrossRef] [PubMed]

13. Lamberg, A.; Nieminen, S.; Qiao, M.; Savilahti, H. Efficient insertion mutagenesis strategy for bacterial genomes involving electroporation of in vitro-assembled DNA transposition complexes of bacteriophage Mu. Appl. Environ. Microbiol. 2002, 68, 705-712. [CrossRef] [PubMed]

14. Pirhonen, M.; Heino, P.; Helander, I.; Harju, P.; Palva, E.T. Bacteriophage T4 resistant mutants of the plant pathogen Erwinia carotovora. Microb. Pathog. 1988, 4, 359-367. [CrossRef]

15. Mohanraj, U.; Kinnunen, O.; Kaya, M.E.; Aranko, A.S.; Viskari, H.; Linder, M. SUMO-based expression and purification of dermcidin-derived DCD-1L, a human antimicrobial peptide, in Escherichia coli. BioRxiv 2018, 343418. [CrossRef]

16. Guzman, L.M.; Belin, D.; Carson, M.J.; Beckwith, J. Tight regulation, modulation, and high-level expression by vectors containing the arabinose PBAD promoter. J. Bacteriol. 1995, 177, 4121-4130. [CrossRef]

17. Leskinen, K.; Tuomala, H.; Wicklund, A.; Horsma-Heikkinen, J.; Kuusela, P.; Skurnik, M.; Kiljunen, S. Characterization of vB_SauM-fRuSau02, a Twort-like bacteriophage isolated from a therapeutic phage cocktail. Viruses 2017, 9, 258. [CrossRef]

18. Gish, W.; States, D.J. Identification of protein coding regions by database similarity search. Nat. Genet. 1993, 3, 266-272. [CrossRef]

19. Soding, J.; Biegert, A.; Lupas, A.N. The HHpred interactive server for protein homology detection and structure prediction. Nucleic Acids Res. 2005, 33, W244-W248. [CrossRef]

20. Chuang, L.-Y.; Cheng, Y.-H.; Yang, C.-H. Specific primer design for the polymerase chain reaction. Biotechnol. Lett. 2013, 35, 1541-1549. [CrossRef]

21. Institute for Molecular Medicine Finland Technology Centre. Sequencing Unit. Available online: https: //www.fimm.fi/en/services/technology-centre/sequencing/ (accessed on 17 June 2019).

22. Kelley, L.A.; Mezulis, S.; Yates, C.M.; Wass, M.N.; Sternberg, M.J.E. The Phyre2 web portal for protein modeling, prediction and analysis. Nat. Protocol. 2015, 10, 845-858. [CrossRef] [PubMed] 
23. Hatfull, G.F.; Pedulla, M.L.; Jacobs-Sera, D.; Cichon, P.M.; Foley, A.; Ford, M.E.; Gonda, R.M.; Houtz, J.M.; Hryckowian, A.J.; Kelchner, V.A.; et al. Exploring the mycobacteriophage metaproteome: Phage genomics as an educational platform. PLoS Genet. 2006, 2, e92. [CrossRef] [PubMed]

24. Kwan, T.; Liu, J.; DuBow, M.; Gros, P.; Pelletier, J. The complete genomes and proteomes of 27 Staphylococcus aureus bacteriophages. Proc. Natl. Acad. Sci. USA 2005, 102, 5174-5179. [CrossRef] [PubMed]

25. Howard-Varona, C.; Roux, S.; Dore, H.; Solonenko, N.E.; Holmfeldt, K.; Markillie, L.M.; Orr, G.; Sullivan, M.B. Regulation of infection efficiency in a globally abundant marine Bacteriodetes virus. ISME J. 2017, 11, 284-295. [CrossRef]

26. Stevens, R.H.; Zhang, H.; Hsiao, C.; Kachlany, S.; Tinoco, E.M.B.; DePew, J.; Fouts, D.E. Structural proteins of Enterococcus faecalis bacteriophage $\varphi$ Ef11. Bacteriophage 2016, 6, e1251381. [CrossRef]

27. Pickard, D.J.J. Preparation of bacteriophage lysates and pure DNA. In Bacteriophages; Springer: Berlin/Heidelberg, Germany, 2009; pp. 3-9.

28. Thomas, J.A.; Quintana, A.D.B.; Bosch, M.A.; De Peña, A.C.; Aguilera, E.; Coulibaly, A.; Wu, W.; Osier, M.V.; Hudson, A.O.; Weintraub, S.T. Identification of essential genes in the Salmonella phage SPN3US reveals novel insights into giant phage head structure and assembly. J. Virol. 2016, 90, 10284-10298. [CrossRef]

29. Yang, H.; Ma, Y.; Wang, Y.; Yang, H.; Shen, W.; Chen, X. Transcription regulation mechanisms of bacteriophages: Recent advances and future prospects. Bioengineered 2014, 5, 300-304. [CrossRef]

30. Miller, E.S.; Kutter, E.; Mosig, G.; Arisaka, F.; Kunisawa, T.; Rüger, W. Bacteriophage T4 genome. Microbiol. Mol. Biol. Rev. 2003, 67, 86-156. [CrossRef]

31. Singh, S.; Godavarthi, S.; Kumar, A.; Sen, R. A mycobacteriophage genomics approach to identify novel mycobacteriophage proteins with mycobactericidal properties. Microbiology 2019, 165, 722-736. [CrossRef]

32. Preston, A. Choosing a cloning vector. In E. coli Plasmid Vectors: Methods and Applications; Casali, N., Preston, A., Eds.; Humana Press: Totowa, NJ, USA, 2003; pp. 19-26.

33. Kutter, E.; Raya, R.; Carlson, K. Molecular mechanisms of phage infection. In Bacteriophages: Biology and Applications, 1st ed.; Kutter, E., Sulakvelidze, A., Eds.; CRC Press: Boca Raton, FL, USA, 2004; pp. 165-222.

34. Khakhum, N.; Yordpratum, U.; Boonmee, A.; Tattawasart, U.; Rodrigues, J.L.M.; Sermswan, R.W. Identification of the Burkholderia pseudomallei bacteriophage ST79 lysis gene cassette. J. Appl. Microbiol. 2016, 121, 364-372. [CrossRef]

35. Shi, Y.; Li, N.; Yan, Y.; Wang, H.; Li, Y.; Lu, C.; Sun, J. Combined antibacterial activity of phage lytic proteins holin and lysin from Streptococcus suis bacteriophage SMP. Curr. Microbiol. 2012, 65, 28-34. [CrossRef] [PubMed]

36. Kinch, L.N.; Ginalski, K.; Rychlewski, L.; Grishin, N.V. Identification of novel restriction endonuclease-like fold families among hypothetical proteins. Nucleic Acids Res. 2005, 33, 3598-3605. [CrossRef] [PubMed] 